La Société suisse de psychiatrie et psychothérapie se réjouit de la publication des lignes directrices portant sur la collaboration entre psychiatres traitants et case managers des assurances. Premier concerné par ces contacts, qui ne peuvent s'établir qu'avec son consentement éclairé, le patient est au centre du dispositif constitué par les employeurs, médecins et assureurs. C'est dans son intérêt que cette collaboration devrait s'instaurer, afin de lui permettre de trouver dans les meilleures conditions possibles la place de travail adaptée à son état de santé.

Dr Pierre Vallon, Président de la SSPP/FMPP

\title{
Coopération entre médecins spécialistes en psychiatrie et psychothérapie et case managers des assureurs privés
}

\author{
Fulvia Rota ${ }^{a}$, \\ Jean-Daniel Sauvant ${ }^{a}$, \\ Renato Marelli ${ }^{b}$, \\ Olivier Ducarrec, \\ Thomas Lack', \\ Bruno Soltermannc \\ a Fédération des médecins \\ psychiatres-psychothéra- \\ peutes de Suisse, FMPP \\ b Société suisse de psychiatrie \\ d'assurance, sspa \\ c Association Suisse d'Assu- \\ rance, ASA
}

Par souci de lisibilité, les termes utilisés dans ce texte sont au masculin, mais ils s'appliquent de manière égale aux hommes et aux femmes.
Correspondance:

Dr Bruno Soltermann

C.-F.-Meyer-Strasse 14

CH-8022 Zurich

Tél. 0442082828

bruno.soltermann[at]svv.ch

Dr. med. Fulvia Rota

SGPP/FMPP

Altenbergstrasse 29

Postfach 686

CH-3000 Bern 8

Tel. 0313138833

Fax 0313138899

sgpp[at]psychiatrie.ch

\section{Introduction}

Les considérations suivantes portent sur les formes de coopération entre les médecins spécialistes en psychiatrie et psychothérapie et les case managers des assureurs privés dans l'intérêt des patients.

Une communication transparente et ouverte au dialogue entre les divers acteurs impliqués accroît la qualité des examens et facilite le processus d'intégration visé.

Thérapie et case management répondent à des mandats différents et indépendants. Cependant, ils peuvent se compléter de manière judicieuse lorsqu'ils sont associés dans l'intérêt du patient pour parvenir à un but défini.

\section{Contacts avec le patient}

Une coopération psychiatre - case manager est recommandée lorsqu'on peut d'un point de vue médical envisager la réinsertion professionnelle d'un patient en situation d'incapacité de travail. Le recouvrement de la capacité de travail et le retour dans le monde du travail sont les buts premiers d'une collaboration centrée sur le patient.

Le traitement psychiatrique-psychothérapeutique a une large visée, qui va bien au-delà de la seule capacité de travail d'un patient. Une coopération entre psychiatre et case manager ne touche donc qu'une partie du traitement psychiatrique.

La façon de la première prise de contact du case manager avec le patient est décisive pour la suite du processus.

En principe, il convient de s'adresser d'abord au psychiatre. Il faut en particulier éviter de téléphoner à la personne assurée sans s'être annoncé au préalable, car une telle prise de contact peut déclencher des sentiments négatifs et renforcer les symptômes de la maladie. Il est pour cette raison fortement recommandé d'annoncer l'appel téléphonique dans une lettre brève et compréhensible.
Il importe en outre d'accorder le plus grand respect à la sphère privée du patient dans le cadre d'un case management. Les entretiens (ainsi que les questionnaires éventuels) ne doivent porter que sur des questions et des informations relatives à l'évaluation et au développement de la capacité de travail dans son contexte social. Il s'agit d'accroître la confiance en soi ainsi que l'autonomie du patient et de promouvoir ainsi sa collaboration active au processus. Le patient doit être informé des contacts entre le psychiatre et le case management.

Si cela s'avère utile, des entretiens peuvent être également convenus entre les différentes personnes impliquées (patient, psychiatre, case manager). Il importe dans ces cas-là de définir clairement le cadre de cette rencontre et de pratiquer une transparence totale en ce qui concerne les informations à disposition. Le patient doit pouvoir prendre connaissance d'une note de dossier ou d'un procès-verbal.

Le case management est présenté de manière plus détaillée dans l'annexe 1. La question des honoraires est traitée dans l'annexe 2 .

\section{Annexe 1: case management $[1,2]$}

\section{Case management}

Le case management est un processus systématique et clairement structuré de gestion d'un cas. Le case manager lance un processus ciblé et prend en charge sur une période déterminée, en accord avec le médecin traitant et l'assurance, la coordination de tous les acteurs impliqués - la personne concernée, les proches parents, le psychiatre, les thérapeutes paramédicaux, l'employeur, les assurances, l'avocat, etc.

Le case manager élabore avec le client/patient et avec les acteurs impliqués des objectifs et fait en sorte qu'ils soient ensuite atteints.

Le case manager fonde son approche sur les ressources du patient, facilite l'accès le plus approprié 
possible aux soutiens disponibles à la personne concernée tout en respectant son désir de prendre en main elle-même son existence.

\section{Le case management dans les assurances}

En cas de prise en charge de personnes accidentées ou malades menacées d'incapacité de travail de longue durée ou même d'invalidité, la plupart des sociétés d'assurance font appel au case management. Certaines disposent de leur propre service, dit interne, de case management, les autres ont recours aux diverses offres externes.

Le case manager, interne et externe, est soumis à la législation sur la protection des données ainsi qu'au devoir de discrétion. Il ne peut se procurer ou transmettre des informations sur la personne concernée que dans les limites légales ou s'il a reçu l'accord par écrit de celle-ci (procuration) après l'avoir informée sur le but, sur l'utilité et sur les conséquences de cette procuration. Une collaboration positive et efficace entre le client/patient et le case manager doit s'appuyer sur une confiance réciproque et sur la transparence.

Les rôles du case manager sont multiples au cours de toute cette phase d'accompagnement: il analyse, utilise et active les ressources et gère l'accès aux prestations en se voulant toujours efficient et efficace dans ses tâches. Il fait office d'intermédiaire et de coordinateur entre la personne concernée et le système de prise en charge et il l'informe et la conseille sur ses droits et obligations. Il veille en outre à ce qu'elle puisse atteindre les buts visés et il l'encourage à faire face par ses propres moyens aux complications, aux conflits, aux difficultés et aux problèmes (empowerment ou «aide à s'aider soi-même») ainsi qu'à élaborer des solutions ou des perspectives d'avenir.

Facteurs déterminants pour un case management La complexité de l'état de santé, de la situation professionnelle, sociale et financière constitue la condition préalable d'un case management, car la personne concernée ne peut pas résoudre seule tous ces problèmes sans un soutien approprié ou sans la mise en œuvre de mesures adaptées aux besoins. En général, la menace d'une incapacité de travail de longue durée pouvant mener à une invalidité est présente. Des études ont établi que les chances de réinsertion diminuent déjà de $50 \%$ au bout de six mois d'incapacité de travail.

Du point de vue de l'assureur lorsque des indemnités journalières ou une rente invalidité sont assurées, le case management a pour objectif le retour au travail ou le maintien avec ou sans adaptation du poste de travail. Il est décisif pour le processus de case management que la personne concernée se montre prête à s'engager dans la voie proposée. Sans l'accord de l'intéressé, il ne portera pas de fruits. Cependant, dans les cas où l'état de santé de la personne concer- née ne permet pas de fixer des objectifs et de prendre des mesures, il peut s'avérer nécessaire de remettre à plus tard (par ex. après un traitement hospitalier) la mise en place éventuelle d'un case management en collaboration avec le médecin traitant.

\section{Coopération entre psychiatre et case manager}

En plus du meilleur traitement possible, le psychiatre, l'employeur et l'assureur doivent avoir pour objectif, dans tous les cas où cela semble possible du point de vue médical, de conjuguer leurs efforts en vue d'une réintégration aussi rapide que possible du patient, employé et assuré, dans le processus de travail ou dans la vie professionnelle. Au traitement psychiatrique s'ajoutent par conséquent une évaluation de la capacité de travail ou de prestation ainsi qu'une appréciation de l'exigibilité. La coopération de chacun des acteurs impliqués, en particulier la collaboration entre le psychiatre et le case manager, joue un rôle décisif. Effectivement, le case manager ne peut se passer de l'appréciation psychiatrique, que ce soit pour être informé de la vulnérabilité du patient lorsqu'il s'agit de prendre contact avec lui ou pour établir, en vue d'une réinsertion professionnelle, la plausibilité de la capacité de prestation, des limitations d'activité suite à une analyse de situation ou l'adéquation de mesures ensuite prévues telles qu'une reprise de travail à l'essai, une augmentation de la capacité de travail, une adaptation de l'activité ou du poste de travail, etc. Il pourra d'autant mieux cibler le processus du case management et de réinsertion professionnelle qu'il connaît à fond la situation médicale souvent complexe du patient et peut ainsi en tenir compte. En contrepartie, le case manager fait office de coordinateur entre toutes les instances impliquées en menant par ex. des entretiens avec le psychiatre ou en convoquant une table ronde. C'est dans ce but qu'a été créée la Coordination de la Gestion de l'Intégration par les Assureurs privés avec les Médecins (CGIAM).

\section{Annexe 2: indemnisation}

CGIAM = Coordination de la Gestion de l'Intégration par les Assureurs privés avec les Médecins [3] Les assureurs privés (accident, indemnités journalières, vie et responsabilité civile) souhaitent promouvoir la gestion de l'intégration et, à cet effet, une intensification des contacts et de la coopération entre spécialistes de l'assurance ou case managers et médecins traitants. Il en résulte naturellement un surplus de travail pour les médecins. C'est pourquoi les assureurs privés ont créé dans le domaine de la LAA des positions tarifaires qui permettent aux médecins traitants de facturer séparément, et non selon TARMED, les entretiens et les tâches de coordination en rapport avec le case management.

Les assureurs privés (indemnités journalières, incapacité de gain ou responsabilité civile) peuvent se 
référer à ces positions tarifaires pour l'indemnisation de prestations.

La CGIAM comprend les positions tarifaires suivantes:

601-CGIAM Case management forfait médecin, première heure

602-CGIAM + case management forfait médecin, par quart d'heure supplémentaire

603-CGIAM Indemnité de déplacement médecin, par tranche de 5 minutes

604-CGIAM Forfait d'urgence médecin ou ACCT

605-CGIAM Certificat détaillé d'incapacité de travail

606-CGIAM Déclaration de capacité de travail pour ACCT, par tranche de 15 minutes

607-CGIAM Etude de dossier, suivi, par tranche de 5 minutes

Les prestations relatives aux positions tarifaires CGIAM sont téléchargeables sur le site Internet de l'Association Suisse d'Assurance www.svv.ch, rubrique médecine $\rightarrow$ formulaires. Elles sont également disponibles en allemand et en italien.

Les positions tarifaires comprennent les entretiens dans le cabinet ainsi que chez l'assureur ou chez l'employeur et l'indemnité de déplacement éventuel. Le tarif inclut également l'étude de dossier dans le cadre de mesures de réinsertion ou l'établissement d'un certificat détaillé d'incapacité de travail. Si la discussion d'un cas doit avoir lieu dans les dix prochains jours ouvrables sur demande de l'assureur, un forfait d'urgence peut être en outre facturé.
Le décompte de toutes ces prestations n'est possible qu'après accord préalable avec l'assureur.

Le document est atteignable pareillement dans les trois langues allemande, française et italienne sur le site Internet de la SSPP (www.psychiatrie.ch) comme celle de l'ASA.

Version française:

www.svv.ch/fr/medecine/service-medical

Version allemande:

www.svv.ch/de/medizin/medizinischer-dienst

Version italienne:

www.svv.ch/de/medizin/in-italiano/servizio-medico

\section{Références}

1 Verein Netzwerk Case Management Schweiz (www.netzwerk-cm.ch).

2 Schnitzer-Brotschi I. Zusammenarbeit Hausarzt Case Manager. Infoméd 2012/1; www.svv.ch

3 Soltermann B. Gestion de l'intégration: CGIAM, ACCT, CIT. Infoméd; 2013/1; www.svv.ch

\section{Sujets d'actualité du forum}

\section{8}

Venez débattre avec nous! Dans la rubrique forum, nous présentons régulièrement des sujets d'actualité politique, économique et scientifique ayant trait au système de santé. Donnez votre avis ou commentez les affirmations de vos confrères. Pour accéder au forum: www.bullmed.ch/forum/ 